\title{
Formación de recursos humanos en Psicología de la Salud a partir de 1959 en Cuba
}

\author{
Formation of human resources in health psychology in Cuba \\ since 1959
}

\author{
I sabel Louro Bernal'; Ada Casal Sosa'"; Libertad Martín Alfonso"'l; Lidia \\ Hernández Gómez "I'; I leana C. Aguilar García'v
}

\author{
'Doctora en Ciencias de la Salud. Investigadora Auxiliar. Escuela Nacional de Salud \\ Pública. La Habana, Cuba. \\ "Especialista en Psicología de la Salud. Profesora Titular. Instituto Superior de \\ Ciencias Médicas de La Habana. La Habana, Cuba. \\ "IIMáster en Psicología de la Salud. Profesora Auxiliar. Escuela Nacional de Salud \\ Pública. La Habana, Cuba. \\ IVMáster en Psicología de la Salud. Profesora Auxiliar. Instituto Superior de Ciencias \\ Médicas de La Habana. La Habana, Cuba.
}

\section{RESUMEN}

El desarrollo de la Psicología de la Salud en Cuba ha constituido un reflejo de las transformaciones de la sociedad con los cambios revolucionarios, la organización del Sistema Nacional de Salud y la maduración del pensamiento y la actuación del psicológo, antecedentes que permitieron introducir el trabajo de este profesional, en instituciones del nivel primario, modificar la concepción de su papel en los hospitales con su inserción, no sólo en psiquiatría, sino en todos los servicios, y ampliar su participación en la atención al proceso salud-enfermedad. Estas condiciones impusieron la necesidad de superación posgraduada y la formación especializada y académica de los profesionales de la psicología, especialmente en la rama de Psicología de la Salud. La formación de recursos humanos en esta disciplina, dentro del sector de la salud, se ha logrado en forma escalonada con la aprobación de tres figuras formativas: la Maestría en Psicología de la Salud en 
1993, la Especialidad en Psicología de la Salud en 1995 y la Licenciatura en Psicología, Mención Salud en el año 2004; procesos que conducen a la existencia de un sistema de formación en Psicología de la Salud de alcance nacional en la Universidad Médica de Cuba.

Palabras clave: Psicología de la Salud, formación de recursos humanos, Cuba.

\begin{abstract}
The development of health psychology in Cuba has reflected the changes occurred in the society due to the revolutionary transformations, the organization of the national health care system and the maturing of the way of thinking and performance of the psychologist. These antecedents allowed introducing the work of this professional into the primary care institutions and modifying the concept of the role of this specialist in the hospital setting through his/her insertion not only in psychiatry but in every service, and extending his/her participation in the care of the health-disease process. The above-mentioned conditions imposed the need of postgraduate education and specialized and academic formation of psychology professionals, particularly in the health psychology branch. The training of these human resources within the health care sector has been gradually carried out thanks to the approval of three formative courses, that is, Master's degree in Health Psychology in 1993; the specialty of Health Psychology in 1995 and Bachelor's degree in Psychology, Health Mention, in 2004. All these processes lead to the existence of a formative system of health psychology nationwide in the Cuban medical university.
\end{abstract}

Key words: Health psychology, human resource formation, Cuba.

\title{
I NTRODUCCI ÓN
}

Antes del triunfo de la Revolución en 1959 la Psicología en Cuba tenía un desarrollo incipiente, era escaso el número de psicólogos dedicados más bien a la práctica clínica de carácter privada y se desempeñaban profesionalmente según la división clásica de la Psicología en cuatro especializaciones: clínica, social, educacional y del trabajo. En el campo de la salud, su papel fundamental era contribuir a establecer el diagnóstico de la enfermedad mental mediante la evaluación psicométrica y a participar en el proceso psicoterapéutico, fundamentalmente del paciente psiquiátrico. El método era básicamente clínico aplicado al diagnóstico y al tratamiento de individuos con trastornos mentales.

Se plantea que el desarrollo de la Psicología de la Salud después del año 1959 constituyó un reflejo sintetizado de cuatro hechos fundamentales: las transformaciones de la sociedad como expresión de los cambios revolucionarios, la organización del Sistema Nacional de Salud (SNS), la maduración del pensamiento psicológico de base materialista y la actividad consecuente de toda una generación de jóvenes psicólogos decididos a encontrarles a su profesión y a su ciencia un 
espacio de plena participación y aporte en la lucha por mejorar el estado de salud del pueblo. ${ }^{1}$

En 1962 se inició la formación masiva de psicólogos en Cuba con la creación de las Escuelas de Psicología en las dos universidades estatales existentes en el momento, debido a la Reforma Universitaria general que llevó a cabo el gobierno revolucionario. Hasta entonces, los pocos psicólogos que había en el país se formaban en universidades privadas o en el extranjero.

Ya en 1966 comenzó a desarrollarse un nuevo perfil profesional para el psicólogo que ejercería sus funciones en el SNS, superando el modelo de la psicología clínica que se limitaba al marco estrecho de la atención psiquiátrica hospitalaria.

Estos antecedentes permitieron la introducción del trabajo del psicólogo en instituciones del nivel primario vinculado al modelo de medicina de la comunidad y posteriormente al modelo de atención integral a la familia. Se modificó la concepción del papel del psicólogo en los hospitales con su inserción en todos los servicios, ya no sólo en psiquiatría. De esta forma se pasó al desarrollo de formas más amplias de conceptualización y participación de la psicología en el proceso salud-enfermedad y en el cuidado de la salud de la población.

La Psicología de la Salud se desarrolló con fuerza en todos los niveles de atención, se desarrollaron departamentos de psicología en todos los hospitales e institutos de investigación en el campo de la salud y con certeza que ese desarrollo se debió en parte a la prioridad que se le dio a este campo del saber en el país, a la sólida formación de un grupo muy activo de experimentados psicólogos y psicólogas en esa área que supo conquistar un espacio profesional dentro del campo de la salud, donde se destacan Lourdes García, Ada Casal, Nereida Calvo, Francisco Morales y Guillermo Ruiz, entre otros. ${ }^{2}$

La emergencia de un nuevo rol profesional para el psicólogo que trabajaría en el SNS de Cuba, debía responder a la necesidad de una vinculación efectiva entre las ciencias sociales y psicológicas, por un lado, y las ciencias naturales, por el otro, generando un conocimiento capaz de brindar una mejor comprensión de la totalidad de condiciones que determinan y participan en el proceso salud-enfermedad en toda su complejidad, así como que contribuya a optimizar la prestación y utilización de los servicios de salud, todo lo cual tributará como consecuencia, niveles más elevados de salud y bienestar para la población. ${ }^{3}$

Se plantean cuatro períodos en la evolución histórica de la Psicología de la Salud (1959-1986), en los que se aprecian especificidades en la búsqueda de solución a los problemas teóricos, organizativos, y metodológicos particulares. El primer período se caracterizó por la aproximación limitada a la salud pública (1959-1965), el segundo se denominó formulación de las premisas teóricas y metodológicoorganizativas de la Psicología de la Salud cubana (1966-1973), el tercero por la franca emergencia de la Psicología de la Salud en el sistema de salud (1974-1984) y el cuarto por el perfeccionamiento y desarrollo de la disciplina (1985-1986). ${ }^{2}$

Posterior a este período se consolidó la integración de la psicología a los diversos servicios de salud y las subsiguientes décadas se destacaron por la participación en la formación de recursos humanos: en los 90 la maestría y la especialidad y en la primera década del 2000, la formación de psicólogos y técnicos de psicología en el seno de las universidades médicas.

Algunos acontecimientos en esta trayectoria tuvieron gran relevancia y contribuyeron al desarrollo de la Psicología en Cuba: la creación del Grupo Nacional 
de Psicología en 1968, con representaciones provinciales, la constitución de la Sociedad Cubana de Psicología de la Salud en 1974, la celebración de congresos de Psicología de la Salud de carácter internacional, las publicaciones periódicas como el Boletín de Psicología y la Revista del Hospital Psiquiátrico de La Habana, así como la elaboración de la proyección estratégica de la disciplina.

La evolución histórica de la Psicología de la Salud refleja las etapas de desarrollo de la Salud Pública Cubana, en las cuales la formación de recursos humanos ha ocupado un rol destacado y el psicólogo de la salud ha contribuido, desde su quehacer profesional, con los resultados que exhibe la salud pública desde el triunfo de la Revolución.

El presente trabajo describe los avances que se han producido en la formación de recursos humanos en el campo de la Psicología de la Salud a partir de 1959, que constituyen hitos de su desarrollo. El método utilizado fue la revisión documental y la sistematización de experiencias de las autoras, algunas protagonistas de los acontecimientos y de la historia descrita.

\section{LA PARTI CI PACI ÓN DE LA PSI COLOGÍ A EN LA FORMACI ÓN DE PROFESIONALES Y TÉCNI COS DE LA SALUD}

En 1966, un pequeño grupo de graduados de Psicología en las universidades cubanas después del triunfo de la Revolución fueron ubicados en el Ministerio de Salud Pública. Desde esa fecha estos profesionales se vincularon a la docencia en la entonces Escuela Nacional de Salud Pública para impartir clases de psicología y temas de ciencias sociales a los médicos y enfermeras que se preparaban para administrar los servicios de salud y realizar actividad de higiene y epidemiología.

En la segunda mitad de los 60 empezó a gestarse un movimiento de inclusión de la psicología en los planes de estudio de técnicos y profesionales de la salud, (médicos, enfermeras, trabajadoras sociales, trabajadores sanitarios, entre otros), y en sus programas de educación continuada, de modo que se favoreciera su desempeño profesional integral, a partir de una valoración de los problemas de salud que considerara los aspectos de la personalidad y el comportamiento del hombre en su condicionamiento socio-cultural y económico. Se considera que la asignatura de Psicología creó las bases de la formación del pensamiento médico social y del enfoque social de la salud en Cuba.

En 1970 aparece el curso de Ciencias de la Conducta ${ }^{4}$ que se diseñó para los técnicos y profesionales que así lo requerían en su formación y se puede considerar que el material de estudio para dicho curso fue el primer libro de texto elaborado por los psicólogos de la salud en Cuba.

Un amplio campo de desarrollo profesional lo constituyó la docencia de psicología en la carrera de medicina y posteriormente en la licenciatura en enfermería y tecnología de la salud.

La creación de la asignatura El hombre y su medio, devenida en Psicología Médica en la carrera de medicina constituyó el inicio de una larga trayectoria de la carrera docente de los psicólogos en el marco de los Institutos de Ciencias Médicas y las Facultades de Medicina, Enfermería y Tecnología de la Salud en Cuba. 
En el año 1972 comenzó la formación de técnicos medios en psicometría para graduados de preuniversitario con una duración de dos años. Al egreso, estos técnicos estaban capacitados para la aplicación de pruebas psicométricas, realización de actividades educativas, observadores de la psicoterapia y en la recogida de datos para las investigaciones, mediante la observación, la entrevista, el cuestionario, a la vez que egresaban capacitados para realizar un análisis estadístico simple.

En 1984, con el surgimiento de la Medicina Familiar y la especialidad médica de Medicina General Integral, se abre un nuevo período de verdadera integración al insertarse el psicólogo como profesor de grupo básico de trabajo y la psicología como una de las cuatro especialidades básicas. El nuevo programa de medicina tuvo entre sus autores un grupo de psicólogos de experiencia en la Atención Primaria de Salud (APS) y en consecuencia contiene un alto porcentaje de temas de psicología. El psicólogo del policlínico desempeña funciones asistenciales en la comunidad y docentes en su propio escenario laboral.

El desafío consistió en que este nuevo especialista debía estar capacitado entre otros propósitos para prestar atención médica integral a niños, adolescentes, mujeres en edad reproductiva y ancianos en el aspecto individual, así como a las familias, las instituciones escolares, fábricas, cooperativas e instituciones de atención a grupos especiales de la sociedad, mediante las acciones de promoción de la salud del individuo, la familia y la comunidad; la prevención específica en individuos sanos y enfermos, la atención a pacientes dispensarizados, y la rehabilitación física, psicológica y social de aquellos que la requieran.

Además de los recursos clásicos de la docencia como la conferencia, el seminario y la observación práctica, el psicólogo participa de las interconsultas, mediante la cual valora con el médico la situación emocional del paciente y/o su familia y ubica el problema de salud en los estilos de vida o comportamientos en salud, valorando la vida familiar, conyugal, laboral, por lo que ello sirve, como recurso importante para que el médico incorpore esa dimensión en la práctica diaria en la atención a las familias y la comunidad.

A partir del curso 2004-2005 la APS, se convierte en el escenario docente por excelencia para la formación de los recursos humanos y el Policlínico Universitario se transforma en una verdadera facultad de ciencias de la salud, por lo que se necesita introducir cambios para lograr la formación de un médico general básico acorde con las necesidades del sistema de salud cubano y de la educación médica superior. ${ }^{5}$

Actualmente en la carrera de tecnología de la salud los contenidos de psicología están distribuidos por los diferentes módulos y su volumen es igual o quizás algo mayor que en la licenciatura de enfermería. ${ }^{6}$

En el nivel secundario se inserta la atención psicológica a pacientes con tratamientos especializados como la rehabilitación cardiovascular, la terapia sexual, trasplantes, entre otros, se incluye además en las actividades docentes de las residencias médicas y cursos de posgrado de aplicación de la psicología ante diversos problemas diagnósticos y terapéuticos.

\section{LA SUPERACIÓN PROFESI ONAL}


Desde la fundación de la Psicología de la Salud y durante los años 60, 70 y 80 la educación de posgrado en esta disciplina se caracterizó por los cursos de superación en el marco de las actividades de educación continuada y de capacitación.

En las décadas de los 70 y los 80 hubo un florecimiento de las actividades de superación profesional organizadas por el Grupo Nacional de Psicología y el Hospital Psiquiátrico de La Habana, a lo que se agrega la concurrencia de especialistas extranjeros de reconocido prestigio. Ejemplos de tales actividades lo constituyen los cursos de educación sexual con Siegfried Schnabl, Mónica Krauss, y de terapia familiar con los profesores Guillermo Bernal y Manuel Gutiérrez. Por esta época el grupo provincial de Ciudad de La Habana convocaba semanalmente a los psicólogos en actividades de superación profesional y actualización en diferentes instituciones del sistema de salud.

Se logró en la década de los 80 convenios para cursos y entrenamientos de posgrado en países del campo socialista, entre ellos, el entrenamiento en terapia sexual y de pareja con el profesor Kratochvil del Hosiptal Psiquiátrico de la Ciudad de Kromierich en Checoslovaquia.

El periodo de crisis económica que sufrió el país durante la década de los 90, incrementó el éxodo de profesionales de la psicología del sector de la salud, aspecto que se debió a la insuficiente remuneración por su categoría, a las negativas de obtención y pago de categorías docentes, a las limitaciones de superación profesional y de condiciones de trabajo, a lo que se agrega las posibilidades más ventajosas que brindaban diferentes campos de aplicación de la psicología en otros sectores de la sociedad.

El psicólogo de la salud continuó cumpliendo funciones asistenciales, docentes, administrativas e investigativas en los diferentes niveles e instituciones del sistema e incluso en misiones internacionalistas.

Las exigencias de las actividades asistenciales que desarrolla el psicólogo en la atención a la familia en épocas de crisis y durante todo el ciclo vital, el aporte en la formación de especialistas médicos y técnicos, la contribución en las tareas de reorientación de la APS, y en la búsqueda de alternativas para frenar el deterioro de la calidad de los servicios de salud apuntan hacia la necesidad de espacios de superación profesional con métodos propios de la psicología.

Aún cuando subsisten problemas que afectan el desempeño profesional de la Psicología de la Salud relacionados con el déficit de psicólogos en el sistema de salud y las insuficientes condiciones y recursos tecnológicos para el diagnóstico y las intervenciones; los psicólogos cubanos han continuado desarrollando la disciplina y las necesidades de superación se incrementaron en la medida que se elevó el nivel científico-técnico en la labor asistencial y se ampliaron los servicios de salud.

Las condiciones antes mencionadas impusieron la necesidad de superación posgraduada y la formación especializada y académica de los profesionales de la psicología, especialmente en la rama de Psicología de la Salud, sin embargo el país contaba con pocos espacios para satisfacer la fuerte demanda de superación que surgía de las necesidades del propio desarrollo de la Psicología, de las exigencias de los problemas que emanaban de la propia práctica profesional, cada vez más diversificada en el sistema de salud. 
El programa de desarrollo de la Psicología de la Salud para el año 2000, planteaba entre sus resultados esperados, que contribuirá a desarrollar los recursos profesionales para la ejecución de acciones psicológicas específicas con el nivel de calificación especializada que se requiere. Para el logro de estas aspiraciones se necesitan programas formativos de alto nivel científico que brinden oportunidad de superación a los profesionales interesados y que generen un potencial de desarrollo al servicio de la Salud Pública Cubana. ${ }^{5}$

\section{FUNDACI ÓN Y DESARROLLO DE LA MAESTRÍ A EN PSI COLOGÍ A DE LA SALUD}

La maestría en Psicología de la Salud se funda en la otrora Facultad de Salud Pública adscrita al Instituto Superior de Ciencias Médicas de La Habana en el año 1993; hecho que constituyó un hito de desarrollo en la formación académica de posgrado para los profesionales de la psicología, ya que este programa fue el primero de su tipo en la disciplina, amplió las posibilidades de acceso a esta modalidad docente y estimuló el desarrollo de la investigación en este campo de conocimiento.

Las tendencias del perfil de salud y enfermedad en la población cubana y problemas en la conducción de servicios de atención a la salud, justifican la existencia de un programa formativo encaminado a la investigación del comportamiento implicado en la salud que contribuya a la solución de problemas de carácter psicológico. De igual forma, el desarrollo del modelo de Psicología de la Salud en Cuba y las necesidades de acción multidisciplinaria que demanda la salud de la población cubana fundamentan la creación de este programa de maestría llamado a fortalecer el trabajo académico, la investigación, el pensamiento epidemiológico y la conciencia de salud colectiva.

Ya desde 1987, el Dr. Reynaldo Pérez Lovelle en su libro "La psiquis en la determinación de la salud", planteaba que el psicólogo requiere una preparación posgraduada teórica y práctica dentro de los marcos del SNS, que la formación en investigación debía ser particularmente fuerte, ya que muchos problemas de esta área están aún por investigar y dilucidar. ${ }^{7}$

Un grupo de psicólogos cubanos de amplia experiencia y trayectoria profesional acometió el diseño curricular del programa de maestría en Psicología de la Salud con la correspondiente fundamentación en el marco de la formación de posgrado en salud pública. Integraron ese grupo figuras destacadas como: Jorge Román Hernández, Reinaldo Pérez Lovelle, Francisco Morales Calatayud, Guillermo Ruiz Rodríguez, Ubaldo González Pérez, Jorge Grau Abalo, Pedro Almirall Hernández, Edelsys Hernández Meléndez, Gema Quintero Danauy, Ada Casal Sosa e Isabel Louro Bernal quien asume junto al Dr. Román la coordinación de la primera edición de la maestría y posteriormente quedó al frente del Comité Académico hasta la actualidad.

Profesores de otras disciplinas contribuyeron al diseño y desarrollo del programa como fueron: Rafael Araujo González, Pablo ResiK Habib, Nereida Rojo Pérez, Leonor Jiménez Cangas, Silvia Martínez Calvo y Héctor Bayarre Vea.

El proceso de fundamentación de la propuesta de la maestría, el diseño curricular y la aprobación en el Ministerio de Educación Superior se caracterizó por períodos de contradicciones de enfoques teóricos y metodológicos, algunos momentos de 
escepticismo ante la viabilidad del programa en tiempos del llamado Período Especial del país y ante la posibilidad de no comprensión de su pertinencia; y otros momentos de gran entusiasmo debido a la oportunidad de desarrollo que se vislumbraba y al apoyo de las autoridades docentes.

Se destacó la elevada motivación del colectivo de profesores dispuestos a asumir el proceso docente, siendo en su mayoría del área asistencial o de otras instituciones docentes como la Facultad de Psicología de la Universidad de la Habana, quienes hicieron esa labor por pura vocación.

La Facultad de Salud Pública, ubicada en los campos de Instituto de Ciencias Básicas y Preclínicas "Victoria de Girón", en la calle 146 y avenida 25, en Cubanacán, municipio Playa, La Habana, contaba con las instalaciones necesarias para el respaldo material del programa, como aulas, biblioteca, laboratorio de computación, sala de video y residencia estudiantil, entre otros recursos indispensables para enfrentar un programa de carácter nacional, de un año de duración y a tiempo completo.

La estructura docente-metodológica de la mencionada Facultad de Salud Pública, su funcionamiento sistemático y riguroso, así como la jerarquización de la actividad y el control sobre los procesos implicados propugnó la fundación, estabilidad y el desarrollo de la maestría.

El plan de estudios de la maestría fue organizado en forma modular y por créditos. En sus inicios y hasta el año 1999 se impartió a tiempo completo y en forma presencial con una duración de un curso académico. Desde el año 2000, con el resurgimiento de la Escuela Nacional de Salud Pública, se plantean nuevas misiones y el programa de maestría se modifica a tiempo parcial en modalidad semipresencial con una duración de dos años.

Los módulos se corresponden con las áreas de la Salud Pública, la Psicología de la Salud, la Metodología de Investigación y la Investigación en diferentes ámbitos de aplicación de la Psicología de la Salud: la comunidad, la familia, el trabajo y el espacio docente. El eje de investigación conduce el diseño del programa siguiendo la lógica del proceso investigativo que culmina en la confección de la tesis de terminación de maestría (TTM).

El enfoque teórico preferencia el paradigma constructivista, por lo que las formas de organización de la enseñanza del proceso docente se apoyan en el carácter participativo y en el trabajo independiente del estudiante.

El perfil del egresado declara que el Máster en Psicología de la Salud estará capacitado para: realizar investigaciones que contribuyan a la solución de problemas de carácter psicológico y social relacionados con la gestión de servicios y la salud de la población; diseñar, aplicar y dirigir proyectos de investigación para el mejoramiento de la salud de la población y el funcionamiento de las instituciones; asesorar instituciones en la gestión de calidad de los servicios a la población y enfrentar científicamente el proceso de formación de recursos humanos en el campo de la salud. ${ }^{8}$

A modo de ilustrar algunos resultados del programa, puede afirmarse que en las evaluaciones realizadas el rendimiento de los estudiantes es alto, el porcentaje de graduados en la edición sobrepasa los estándares previstos, el nivel de satisfacción de los egresados es elevado, y las variables del sistema de evaluación y acreditación de maestrías tuvieron un buen comportamiento, al punto que en el año 
2004 el programa obtuvo la condición de Excelencia en la evaluación externa de la Junta de Acreditación Nacional de Cuba.

En materia de resultados científicos asociados a la maestría, en el año 2000 se realizó un estudio descriptivo retrospectivo a partir del análisis de contenido de los trabajos de TTM de los egresados.

Fueron identificadas 5 grandes áreas temáticas en cuanto a la producción científica: producción tecnológica, investigaciones en servicios y sistemas de salud, riesgo psicológico, calidad de vida y salud mental.

Los resultados más destacados de investigación en la maestría se encuentran en el área denominada producción de tecnología evaluativa, mediática y metodológica, los cuales se resumen en la confección y validación de pruebas o cuestionarios para la medición del fenómeno subjetivo, la confección y validación de materiales educativos como manuales, videos y mensajes fílmicos de salud. En investigaciones en servicios de salud, se destacan las evaluaciones de la satisfacción de la población con modalidades tales como: ingreso en el hogar, círculos de abuelos, atención ambulatoria a enfermos VIH/SIDA.

En relación con la evaluación al personal de salud y prestadores de servicios, sobresalen los estudios del estrés laboral asistencial en personal de enfermería oncológica, médico-quirúrgica y en médicos y enfermeras de familia. Se realizó evaluación de desempeño del psicólogo en la APS, en hospitales pediátricos y en la atención a la familia. Se describieron las contribuciones cubanas a la Psicología de la Salud en el periodo 1966-98 y se evaluó la asignatura Psicología Médica de la carrera de medicina y de enfermería.

Se realizó la caracterización de la calidad de vida en términos socio-psicológicos en: pacientes ulcerosos, hipertensos, asmáticos, con retinopatía, en niños sordos, enfermos por cáncer y SIDA, así como en ancianos, mujeres y adolescentes.

Se identificaron factores psicosociales de riesgo en: mujeres, embarazadas de alto riesgo, adultos mayores, adolescentes, pacientes deprimidos, conducta suicida. Fueron realizados además estudios de estrés, moduladores psicológicos y apoyo social enfocados al cáncer, la hipertensión, la depresión, el dolor crónico, la violencia intrafamiliar, el alcoholismo y la conducta suicida. ${ }^{9}$

En resumen, la maestría ha constituido el marco de desarrollo de líneas temáticas de investigación mediante proyectos que han sido de interés al sistema de salud, y que han generado temas de doctorado como son: la salud de la familia, la adherencia terapéutica en hipertensos y el estrés laboral asistencial.

\section{ESPECI ALI ZACIÓN EN PSI COLOGÍ A DE LA SALUD}

En 1995 se creó la Especialización en Psicología de la Salud, una nueva forma de posgrado (aprobada por el Ministerio de Educación Superior, Res. 10/95) que proporciona conocimientos teóricos profundos y propicia una práctica especializada que, en correspondencia con los objetivos del SNS, permite a estos profesionales cumplir con las acciones de promoción, prevención, diagnóstico, tratamiento y rehabilitación psicológicas, propias de esta disciplina. Esta especialización tiene como objetivo central formar modos de actuación profesional, por lo que desarrolla competencias que permiten lograr un desempeño exitoso en estas acciones. 
La formación del especialista que requiere el SNS debe estar basada en la práctica en las propias áreas de trabajo de las instituciones, bajo la supervisión y tutoría de aquellos profesionales que tengan la calificación y la experiencia requerida.

Los contenidos del programa de formación incluyen los conocimientos, así como las habilidades y hábitos, las actitudes y convicciones que conforman los métodos de trabajo de los especialistas de Psicología de la Salud. El educando se apropia de cada uno de ellos mediante la realización y sistematización de acciones en el ejercicio de la profesión de psicólogo del SNS.

El diseño curricular del programa estuvo asesorado por la Dirección de Especialización del MINSAP en la persona de la metodóloga Máster Nidia Nolla Cao, quien tuvo una participación destacada en todo lo concerniente al programa. El plan de estudio se concibió en forma modular y su estructura responde a la lógica interna de la ciencia y a la organización del servicio donde el educando desarrollará sus actividades. Se tuvieron en cuenta principios pedagógicos reguladores del proceso docente-educativo como son: la combinación del estudio-trabajo, la enseñanza tutorial y el estudio-trabajo independiente del educando.

El modelo del especialista plantea que el psicólogo especializado en Psicología de la Salud es un profesional de perfil amplio, competente para desempeñar funciones de atención psicológica, asesoramiento, investigación, docencia y administración, en la solución de situaciones psicológicas, psicosociales y psicofisiológicas que presenten individuos, familias, grupos y colectivos en las diferentes unidades y niveles del SNS, en los que realizan acciones de promoción, prevención, recuperación y rehabilitación psicológica. ${ }^{10}$

Este modelo incluye la caracterización del especialista y el perfil profesional. La caracterización expresa las cualidades éticas y morales que deben cumplir los futuros especialistas. El perfil profesional determina los objetivos generales a lograr por los especialistas al término de su formación.

En la caracterización del especialista consta que este profesional debe desarrollar su actividad sobre la base de la aplicación del método científico, desarrollar una actitud humanista y solidaria incorporada a su comportamiento profesional cotidiano, con elevado espíritu crítico y autocrítico, desarrollar el espíritu de auto superación constante en el plano técnico, científico, filosófico, ideológico y cultural; influir con su trabajo en los cambios conductuales que le permitan al individuo lograr una calidad de vida superior, con el debido respeto a la decisión personal, con plena conciencia de que la responsabilidad de la vida y la felicidad de cada persona es tanto de ella como del especialista; tomar conciencia de la responsabilidad que se adquiere al enfrentarse a un ser humano que sufre y que confía en que se va a aliviar con la ayuda del especialista, así como mantener las normas de la ética vigentes para la atención a pacientes o grupos.

Para lograr los objetivos generales del plan de estudios, planteados en el modelo del especialista, se estructuraron cinco áreas del conocimiento y 28 módulos, además de cursos y estancias; con una duración general de dos años. Para la función de Atención Psicológica, se han definido las cinco áreas del conocimiento con 24 de los módulos y las dos estancias, para las funciones restantes un módulo cada una y los cursos complementarios. El diseño tiene un total de $4224 \mathrm{~h}$ de las cuales la mayoría está dedicada a actividades de entrenamiento.

Los módulos y sus contenidos han sido organizados de manera que se logren y perfeccionen las capacidades de diagnóstico y tratamiento en el primer año y las de tratamiento y rehabilitación en el segundo. 
Cada módulo está precedido de un objetivo que expresa el sistema de habilidades y conocimientos, así como las técnicas y métodos de trabajo. Estos sistemas de habilidades y conocimientos están encaminados también a la formación de las actitudes y convicciones necesarias para conformar los métodos de trabajo de los especialistas de Psicología de la Salud.

Desde el comienzo de la especialidad en el curso 1995-96, hasta el presente año 2008, se han impartido ocho ediciones. Se han graduado aproximadamente 150 psicólogos, de casi todas las provincias del país. En investigación realizada en el curso 2000-2002 para evaluar los resultados del programa de especialización, se constató un alto grado de compromiso y de satisfacción entre los educandos, tutores y profesores, se observó el impacto positivo en la población atendida por los especialistas, y entre los decidores y otros profesionales de la salud.

Actualmente la especialidad está en etapa de reestructuración y adecuación, ya que el surgimiento de la carrera de Licenciatura en Psicología, Mención Salud hace necesario modificar aspectos del programa que ya se imparten en el pregrado, y dirigir el diseño hacia objetivos más específicos.

\section{LA CARRERA DE LI CENCI ATURA EN PSI COLOGÍ A, MENCIÓN SALUD}

En el curso 2004-2005, se inició en Cuba la carrera de Licenciatura en Psicología, Mención Salud auspiciada por el Ministerio de Salud Pública y los Centros de Educación Médica Superior.

La fundamentación de la carrera, su diseño curricular, la conciliación con las facultades de psicología, la aprobación a nivel del MINSAP, los institutos de ciencias médicas y el Ministerio de Educación Superior, fue un arduo proceso de trabajo de profesionales de vasta experiencia bajo la coordinación del Grupo Nacional de Psicología.

El surgimiento de esta formación en Psicología de la Salud en el marco de la Universidad Médica respondió a la necesidad de incrementar los recursos humanos de nivel técnico y profesional dedicados a esta disciplina, que ya eran escasos en el sector de la salud, a lo que se agrega el limitado número de ingreso al sector proveniente de los graduados de las facultades de psicología. Por otra parte, ya se había identificado la necesidad de un enfoque más sólido en la formación en Psicología de la Salud, más dirigido a la práctica en los diferentes servicios de psicología de las instituciones de salud y orientados no sólo a la curación, sino a la promoción, la prevención, la acción organizacional, comunitaria y familiar.

La existencia de una masa crítica de especialistas y másteres en Psicología de la Salud, la consolidación del modelo profesional en salud que alcanza los 40 años en Cuba, unido a la madurez que ha adquirido la psicología como ciencia y profesión a nivel mundial, constituyen antecedentes de este hecho crucial en el país, que puede considerarse un logro de la formación de recursos humanos en salud.

El desarrollo científico-técnico contemporáneo requiere que la psicología en el sector de la salud mantenga un lugar de avanzada, que progrese de forma armónica con el desarrollo de la salud pública, que sus profesionales contribuyan a que las personas y las familias reciban servicios de calidad, más humanizados e integrales que coadyuven al mejoramiento de la salud y al disfrute pleno de la vida. 
La característica peculiar de este proceso formativo es la aplicación del nuevo modelo pedagógico en el diseño curricular y de esta manera se alcanzan tres tipos de graduados, en forma escalonada con niveles crecientes de complejidad.

El primer año de la carrera se cursa solamente con actividades académicas que permiten la calificación de Técnico Básico en Psicología. En segundo año, los educandos se insertan como técnicos, bajo la orientación de un tutor, en una institución de salud debidamente acreditada, donde exista un servicio de psicología para la atención a la población y alternan sus actividades laborales con encuentros teóricos cada semana. Al término del 6to. semestre, se califica como Técnico Medio Superior en Psicología, (psicometrista), el 7 mo. semestre se dedica a las actividades laborales relativas al perfil de Técnico Medio Superior. Los semestres 8 vo., 9no. y $10 \mathrm{mo}$. son de práctica pre profesional y ejercicios de terminación de estudios superiores, hasta obtener el título de Licenciado en Psicología. ${ }^{11}$

El nuevo modelo pedagógico se basa en la educación en el trabajo, es flexible y permite adaptarse a situaciones sociales, laborales e individuales de los estudiantes y a las particularidades territoriales. Es un modelo centrado en el estudiante, estructurado para favorecer la organización y estimular el proceso del aprendizaje durante toda la carrera. ${ }^{12}$

A diferencia del modelo pedagógico tradicional centrado en el profesor, bajo este nuevo modelo el estudiante asume activamente su proceso de formación siendo el principal gestor de su propio conocimiento, donde la adquisición de habilidades y el aprendizaje se producen en su propio puesto de trabajo, lo que permite la integración de los ejes docentes, asistenciales y de investigación.

Con su aplicación se logra el fomento de la interdisciplinariedad y la transdisciplinariedad frente al aislamiento disciplinario, la promoción de métodos activos de enseñanza, la solución de problemas, y el trabajo independiente supervisado. Se promueve la realización por los educandos de investigaciones de complejidad creciente de los principales problemas identificados. Se potencia la preparación del estudiante para aprender por sí mismo en forma activa y permanente, fomentándole las capacidades para la obtención de información, la observación objetiva el razonamiento lógico y el juicio crítico. ${ }^{11}$

El nuevo modelo pedagógico en la formación técnica y de pregrado en Psicología de la Salud promueve la formación de profesionales con un alto grado de compromiso con su pueblo y de humanidad, gran vocación de servicios y sentido ético en su actuación, una concepción integral de la Psicología como ciencia social y biológica, desarrollo de un pensamiento científico, ya que parte de los problemas y necesidades relevantes de salud, para promover soluciones pertinentes a través del desarrollo de las actividades formativas en escenarios reales de los servicios, en los distintos espacios formativos del SNS.

Primeramente la carrera comenzó en siete Centros de Educación Médica Superior del país, Ciudad de La Habana, Matanzas, Villa Clara, Cienfuegos, Ciego de Ávila, Camagüey y Santiago de Cuba. En el actual curso 2008-2009 la carrera se está impartiendo en todo el país y en la matrícula están representados la totalidad de los municipios.

Constituyen retos para los Centros de Educación Médica Superior el aseguramiento que requiere la formación de nivel superior que genere un profesional de perfil amplio, comprometido, con alto sentido ético en la atención a la cultura y sistema de valores del paciente, la familia y la comunidad, que brinde servicios tomando en cuenta la unidad biopsicosocial del ser humano, lo que logra a través de una sólida 
formación científica que le permita acceder a distintas opciones de trabajo y emprender estudios de posgrado tendentes a su educación permanente, así como una actitud de aprendizaje y vinculación con su práctica profesional con la docencia y la investigación (Fernández Sacasas J, Diego Cobelo J. Tendencias contemporáneas en Educación Médica Superior. Presentación Consejo de Rectores y Decanos. La Habana 2004).

Como resultado de la referida trayectoria de fundación y desarrollo de la Psicología de la Salud en Cuba y de la aprobación de un nuevo perfil profesional, se cuenta con formulaciones conceptuales en las que se plantea:

La Psicología de la Salud es la rama aplicada de la Psicología que se dedica al estudio de los componentes subjetivos que intervienen en el proceso saludenfermedad y en la atención a la salud. Se ocupa de estudiar aquellos procesos de carácter psíquico que participan en la determinación del estado de salud, en el riesgo de enfermar, en la situación de enfermedad y en la recuperación, así como estudia las circunstancias interpersonales que se ponen de manifiesto en la prestación de servicios de salud. ${ }^{13}$ En tanto modelo profesional, contempla un espectro amplio de actividad que incluye acciones de promoción de salud, prevención de las enfermedades, atención a los enfermos, a las personas con secuelas y así como aquellas que se encuentran en fase terminal, al tiempo que la dinámica y funcionamiento de las organizaciones prestadoras de servicios de salud es también objeto y campo de proyección de la Psicología de la Salud.

La Psicología en su contribución al cuidado de la salud en Cuba definió los siguientes objetivos básicos: contribuir al desarrollo óptimo de la personalidad a través de todo el ciclo vital para promover la salud, el bienestar psicológico y la capacidad de rendimiento del individuo, contribuir a la integralidad de la atención en salud al incorporar la valoración de los aspectos psicológicos del hombre sano y enfermo en las acciones de promoción, diagnóstico, tratamiento y rehabilitación, contribuir a la óptima prestación y utilización de los servicios de salud mediante la valoración de los aspectos socio-psicológicos involucrados.

\section{CONSI DERACI ONES FI NALES}

En el campo de la formación de recursos humanos en Psicología de la Salud se logró en forma escalonada, dentro del sector de la salud, la aprobación de tres figuras: la Maestría en Psicología de la Salud en 1993, la Especialidad en Psicología de la Salud en 1995 y la Licenciatura en Psicología, Mención Salud en el año 2004, procesos que conducen a la existencia de un sistema de formación en Psicología de la Salud de alcance nacional en los Centros de Educación Superior de Cuba.

La Maestría en Psicología de la Salud, la Especialidad en Psicología de la Salud y la Licenciatura en Psicología, Mención Salud, constituyen hitos del desarrollo de la Psicología de la Salud en Cuba que expresan la consolidación de la psicología en el área de formación de recursos humanos en salud, y un paso de avance en el posicionamiento del psicólogo en el sector y de la psicología en la sociedad al servicio de la salud de la población.

Se requiere velar por las condiciones que garanticen la estabilidad y calidad de la formación de recursos humanos de alta calificación profesional y técnica en este campo de actuación del psicólogo, de significativa relevancia entre los campos de aplicación de la psicología, al dedicarse al fomento de la salud y la recuperación de la enfermedad y sus secuelas. 


\section{REFERENCI AS BI BLI OGRÁFICAS}

1. Grupo Nacional de Psicología. Programa de desarrollo 2000. Psicología de la Salud. La Habana: Editorial Ciencias Médicas; 2001.

2. González RF. La Psicología en Cuba: un relato para su historia 1960- 1990. Rev Interamericana Psicología. 2000;34(2): 185-198.

3. García AL, Rodríguez AL. La psicología de la salud en Cuba. Papeles del Psicólogo. 1983; Septiembre, № 10 y № 11.

4. Grupo Nacional de Psicología. Ciencias de la Conducta. La Habana: MINSAP; 1970.

5. Navas CM. Proyecto Policlínico Universitario. Reflexiones de su implementación en el Policlínico Universitario. [publicado 21 Sep 2007, citado 18 Sep 2008]. Disponible en:

http://www.portalesmedicos.com/publicaciones/articles/691/1/Proyecto-PoliclinicoUniversitario-Re flexiones-de-su-implementacion-en-el-Policlinico-UniversitarioSanta-Clara.html

6. Pérez LR. La psicología de la salud en Cuba. Psicología Científica. [citado 18 Sep 2008]. Disponible en: http://www.psicologiacientifica.com/bv/psicologia-112-1-lapsicologia-de-la-salud-en-cuba.html

7. Pérez Lovelle R. La psiquis en la determinación de la salud. La Habana: Editorial Científico Técnica; 1987.

8. Programa de Maestría en Psicología de la Salud. La Habana: Escuela Nacional de Salud Pública, MINSAP; 2001.

9. Autoevaluación del Programa de Maestría en Psicología de la Salud. La Habana: Escuela Nacional de Salud Pública, MINSAP;2003.

10. Casal Sosa A. Plan de Estudios de la Especialización Posgraduada en Psicología de la Salud. La Habana: Instituto Superior Ciencias Médicas de La Habana, ViceMin. Docencia; 1997.

11. Plan de estudio de la carrera de Psicología, Mención Salud. Facultad de Psicología. La Habana: Universidad de La Habana, Instituto Superior Ciencias Médicas de La Habana;2004.

12. Fernández Sacasas J. Educación Médica Superior. Realidades y Perspectivas a las puertas del nuevo siglo. Material Bibliográfico de la Maestría en Educación Médica. La Habana: Escuela Nacional de Salud Pública, MI NSAP; 1999.

13. Morales Calatayud F. Psicología de la Salud. Conceptos básicos y proyecciones de trabajo. La Habana: Editorial Científico Técnica; 1999. 
Recibido: 6 de octubre de 2008.

Aprobado: 21 de octubre de 2008.

I sabel Louro Bernal. Escuela Nacional de Salud Pública. Calle Línea esq. I. El Vedado 10400. La Habana, Cuba. 